\title{
Stuck Between a Rock and a Hard Place: The Clinical Conundrum of Managing Cardiac Surgical Patients During the SARS-CoV-2 Pandemic
}

\author{
Nitish Dhingra ${ }^{1}$, Subodh Verma ${ }^{1}$, Terrence Yau ${ }^{2}$, Bobby Yanagawa ${ }^{1}$, and Makoto Hibino ${ }^{1}$ \\ ${ }^{1}$ University of Toronto \\ ${ }^{2}$ Toronto General Hospital
}

October 18, 2021

\begin{abstract}
Deferring non-emergent cardiac surgery became the strategy of choice for several international healthcare systems afflicted by high case burdens of severe acute respiratory syndrome coronavirus 2 (SARS-CoV-2/COVID-19) in order to both conserve valuable healthcare resources and protect patients from possible exposure. Missing from the available dataset to help guide policy development has been a clear understanding of the extent to which COVID-19 infection modulates cardiac surgery outcomes. In their investigation, Bonalumi and colleagues uncovered an inpatient COVID-19 positivity rate of almost 10 times higher than that of the general Italian population, as well as a mortality rate over 20 times higher amongst cardiac surgery patients with perioperative COVID-19 infection compared to those COVID-negative. While the summation of available evidence points to the serious consideration cardiac surgeons must give to delaying surgeries during the COVID-19 pandemic, recognition must be given to the risks that postponing cardiac surgery may have on patient outcomes. Emerging data is beginning to demonstrate the efficacy of vaccination in preventing postoperative COVID-19 infection and morbidity.
\end{abstract}

Stuck Between a Rock and a Hard Place: The Clinical Conundrum of Managing Cardiac Surgical Patients During the SARS-CoV-2 Pandemic

\section{Running Head: Cardiac Surgery During the SARS-CoV-2 Pandemic}

Nitish K. Dhingra ${ }^{1}$, Subodh Verma ${ }^{1}$, Terrence M. Yau ${ }^{2}$, Bobby Yanagawa ${ }^{1}$, Makoto Hibino ${ }^{1}$

${ }^{1}$ Division of Cardiac Surgery, St. Michael's Hospital, University of Toronto, Toronto, Canada

${ }^{2}$ Division of Cardiac Surgery, University Health Network, University of Toronto, Toronto, Canada

Corresponding Author

Makoto Hibino MD MPH PhD, Division of Cardiac Surgery, St. Michael's Hospital, University of Toronto, 30 Bond Street, Toronto, Ontario, M5B 1W8, Canada.

TEL 416864 5997; FAX 4168645881

mhibino-ngy@umin.org

\section{Abstract}

Deferring non-emergent cardiac surgery became the strategy of choice for several international healthcare systems afflicted by high case burdens of severe acute respiratory syndrome coronavirus 2 (SARS-CoV2/COVID-19) in order to both conserve valuable healthcare resources and protect patients from possible exposure. Missing from the available dataset to help guide policy development has been a clear understanding 
of the extent to which COVID-19 infection modulates cardiac surgery outcomes. In their investigation, Bonalumi and colleagues uncovered an inpatient COVID-19 positivity rate of almost 10 times higher than that of the general Italian population, as well as a mortality rate over 20 times higher amongst cardiac surgery patients with perioperative COVID-19 infection compared to those COVID-negative. While the summation of available evidence points to the serious consideration cardiac surgeons must give to delaying surgeries during the COVID-19 pandemic, recognition must be given to the risks that postponing cardiac surgery may have on patient outcomes. Emerging data is beginning to demonstrate the efficacy of vaccination in preventing postoperative COVID-19 infection and morbidity.

\section{Manuscript}

The relationship between severe acute respiratory syndrome coronavirus 2 (SARS-CoV-2/COVID-19) infection and cardiovascular disease is complex. SARS-CoV-2 has cardiovascular sequelae and patients with established cardiovascular disease are at risk of higher rates of infective morbidity and mortality. ${ }^{1}$ In an attempt to preserve scarce healthcare resources for COVID-19 patients, several international healthcare systems adopted a strategy of deferring non-emergent cardiac surgeries during the pandemic, and particularly during times of higher case burdens. ${ }^{2,3}$ Specific recommendations advising against performing non-emergent cardiac operations amongst patients with acute viral infections have also been made. ${ }^{2}$ As a result, an international survey of global cardiac surgical centres in 2020 showed a median reduction in case volume of $50-75 \%$, with $5 \%$ of centres cancelling even emergency cases. ${ }^{4}$ Missing from current available data to help inform policy development and implementation, however, has been a robust understanding of the exact impact of SARS-CoV-2 infection on cardiac surgical outcomes.

Bonalumi and colleagues probed rates of in-hospital mortality amongst adult cardiac surgical patients at 22 Italian centres from February to May of $2020 .{ }^{5}$ Of the 1354 patients analyzed, a total of $48(3.5 \%)$ were documented to be positive for COVID-19 in the perioperative period. Unsurprisingly, the authors identified a significantly higher in-hospital mortality amongst COVID-19 positive patients compared to their COVID-negative counterparts $(20.8 \%$ vs. $0.9 \%, \mathrm{p}<0.001)$. Importantly, despite a trend towards higher rates of emergent surgery amongst the COVID-19 positive group ( $6.3 \%$ vs. $0.3 \%, \mathrm{p}=0.11)$, an independent association of COVID-19 infection and in-hospital mortality was maintained in multivariable analysis. The authors also identified low oxygen saturation and increasing age as independent risk factors for in-hospital mortality within the COVID-19 positive subgroup. Finally, the authors reported an inpatient COVID-19 positivity rate almost 10 times higher than that of the general population of Italy at the time. Given that $77 \%$ of these positive tests were uncovered postoperatively, this raises the possibility that either preoperatively infected patients were tested before becoming PCR-positive, or that healthcare facilities acted a vehicle for transmission. The results presented by Bonalumi and colleagues are similar to those reported in other surgical services amongst COVID-19 patients in Italy ${ }^{6}$, as well as analyses of cardiac surgical patients with COVID19 at other centres. ${ }^{7-9}$ The summation of the available evidence points towards the serious consideration clinicians must give to deferring non-emergent cardiac surgery of COVID-19 patients, particularly amongst those with advanced age or sub-optimal pulmonary function.

While necessary for achieving both the broader public health priorities and protecting patients with operable cardiovascular disease, it is important to recognize that decisions to defer cardiac surgery may have unintended negative consequences. Literature published prior to the SARS-CoV-2 pandemic demonstrated that mortality rates may increase by $11 \%$ every month amongst patients waiting for coronary artery bypass grafting. ${ }^{10}$ This figure becomes far more salient when it is understood that a potential range of 1-8 months may be required to clear the cardiac surgical backlog created by the COVID-19 pandemic. ${ }^{11}$

Clinicians today must choose between expending valuable healthcare resources, as well as exposing patients to potential SARS-CoV-2 infection, or allowing for an increasing burden of cardiovascular disease to continue to afflict already high-risk patients. A number of solutions have been offered to address this, including the development of clinical models to aid in triaging the patients most likely to suffer adverse events while on the cardiac surgery waitlist, ${ }^{12}$ as well as the implementation of meticulous public health measures including infection control and isolation. ${ }^{13}$ Perhaps no solution has been more promising, however, than COVID- 
19 vaccination. A recent analysis of over 10,000 surgical patients demonstrated a significant reduction in postoperative COVID-19 infections, as well as pulmonary and thrombotic complications, amongst those vaccinated against COVID-19 compared with those unvaccinated. ${ }^{14}$

In summary, Bonalumi and colleagues have provided further evidence that confirms the significant additional risk perioperative COVID-19 infection confers to patients undergoing cardiac surgery. While balancing the risk of COVID-19 infection with the risk of deferring major cardiovascular surgery remains a dilemma for surgeons internationally, emerging evidence suggests that the solution may lie in continuing advocacy and education surrounding COVID-19 vaccination.

\section{References}

1. Aghagoli G, Gallo Marin B, Soliman LB, Sellke FW. Cardiac involvement in COVID-19 patients: Risk factors, predictors, and complications: A review. J. Card. Surg. 2020;35:1302-1305.

2. Hassan A, Arora RC, Adams C, Bouchard D, Cook R, Gunning D, et al. Cardiac Surgery in Canada During the COVID-19 Pandemic: A Guidance Statement From the Canadian Society of Cardiac Surgeons. Can. J. Cardiol. 2020;36:952-955.

3. Haft JW, Atluri P, Ailawadi G, Engelman DT, Grant MC, Hassan A, et al. Adult cardiac surgery during the COVID-19 pandemic: A tiered patient triage guidance statement. J. Thorac. Cardiovasc. Surg.2020;160:452-455.

4. Gaudino M, Chikwe J, Hameed I, Robinson NB, Fremes SE, Ruel M. Response of Cardiac Surgery Units to COVID-19: An Internationally-Based Quantitative Survey. Circulation.2020;142:300-302.

5. Bonalumi G. Prognostic value of SARS-CoV-2 on patients undergoing cardiac surgery. J. Card. Surg. 2021.

6. Doglietto F, Vezzoli M, Gheza F, Lussardi GL, Domenicucci M, Vecchiarelli L, et al. Factors Associated With Surgical Mortality and Complications Among Patients With and Without Coronavirus Disease 2019 (COVID-19) in Italy. JAMA Surg. 2020;155:691-702.

7. Fattouch K, Corrao S, Augugliaro E, Minacapelli A, Nogara A, Zambelli G, et al. Cardiac surgery outcomes in patients with coronavirus disease 2019 (COVID-19): A case-series report. J. Thorac. Cardiovasc. Surg. 2020.

8. Barkhordari K, Khajavi MR, Bagheri J, Nikkhah S, Shirzad M, Barkhordari S, et al. Early respiratory outcomes following cardiac surgery in patients with COVID-19. J. Card. Surg.2020;35:2479-2485.

9. Cardiothoracic Interdisciplinary Research N, Collaborative CO. Early outcomes and complications following cardiac surgery in patients testing positive for coronavirus disease 2019: An international cohort study. J. Thorac. Cardiovasc. Surg. 2021;162:e355-e372.

10. Rexius H, Brandrup-Wognsen G, Oden A, Jeppsson A. Mortality on the waiting list for coronary artery bypass grafting: incidence and risk factors. Ann. Thorac. Surg. 2004;77:769-774; discussion 774-765.

11. Salenger R, Etchill EW, Ad N, Matthew T, Alejo D, Whitman G, et al. The Surge After the Surge: Cardiac Surgery Post-COVID-19.Ann. Thorac. Surg. 2020;110:2020-2025.

12. Sun LY, Eddeen AB, Wijeysundera HC, Mamas MA, Tam DY, Mesana TG. Derivation and validation of a clinical model to predict death or cardiac hospitalizations while on the cardiac surgery waitlist. CMAJ. 2021;193:E1333-E1340.

13. Farrington WJ, Robinson NB, Rahouma M, Lau C, Hameed I, Iannacone EM, et al. Cardiac Surgery Outcomes in an Epicenter of the COVID-19 Pandemic. Semin. Thorac. Cardiovasc. Surg. 2021.

14. Prasad NK, Lake R, Englum BR, Turner DJ, Siddiqui T, Mayorga-Carlin M, et al. COVID-19 Vaccination Associated with Reduced Post-Operative SARS-CoV-2 Infection and Morbidity. Ann. Surg.2021. 